\title{
Investigation on the AC breakdown voltage of RBDPO Olein
}

\begin{abstract}
Vegetable oil is one of the alternatives for mineral oil as dielectric insulating fluid in transformers. Vegetable oil is environmentally friendly, it is biodegradable, it is renewable and has high flash point which ensure more safety for in-service operation. There are many types of vegetable oils and one of them is palm based oil. Currently, there are different types of palm based oils were considered for transformers application such as Crude Palm Oil (CPO), Palm Kernel Oil (PKO), Refined, Bleached and Deodorized Palm Oil (RBDPO), Palm Fatty Acid Ester (PFAE) and PKO Alkyl Ester. In this paper, a study is carried out to investigate the AC breakdown voltage of RBDPO Olein before and after dried procedure. The study shown that the AC breakdown voltage have increased significantly after various samples of RBDPO Olein being dried. However, fats and vitamin E contents in each samples demonstrated indefinite effects to the AC breakdown voltage for both conditions.
\end{abstract}

Keyword: AC breakdown voltage; Palm based oil; Transformers 